\title{
VOLATILIDADE, MAGNITUDE DOS PROVENTOS E A SINALIZAÇÃO NA POLÍTICA DE DISTRIBUIÇÃO DE LUCROS
}

\author{
Volatility, dividend yield and signaling theory through \\ payout policy
}

\author{
José de Pietro Neto ${ }^{1}$ \\ Oscar Claudino Galli² \\ Roberto Frota Decourt ${ }^{3}$
}

\begin{abstract}
Resumo
Este trabalho procurou testar a teoria da sinalização por meio das notícias veiculadas aos investidores que se referem ao anúncio da distribuição de proventos de 1998 a 2006, para as ações integrantes da carteira teórica do Ibovespa de janeiro a abril de 2006. Para a realização deste artigo, foram efetuados cálculos para verificar a presença de retornos anormais nas datas de anúncio da distribuição de juros sobre capital próprio, dividendos e pagamento simultâneo. Adicionalmente, foram realizados testes nos dias ao redor da data de anúncio, numa janela de 11 dias, incluindo a data da divulgação do pagamento dos proventos na busca de vazamento de informações e para verificar a possibilidade de arbitragem na janela em estudo. Os testes realizados consideraram a presença de retornos anormais e acumulados quando o mercado estava operando em níveis diferentes de volatilidade do mercado e de magnitude do provento anunciado, procurando verificar se os investidores interpretam e penalizam o pagamento de dividendos de maneira simétrica com relação às variações de volatilidade do mercado e de magnitude do provento anunciado. Os resultados evidenciaram a presença de retornos anormais significativos.
\end{abstract} Dividendos.

Palavras-chave: Volatilidade; Política de Dividendos; Teoria da Sinalização;

\footnotetext{
${ }^{1}$ Doutorando, Universidade de Brasília (UnB), CLN 404, Asa Norte, 10 - CEP70847-550, Brasília, DF. E-mail: jpietron@gmail.com

2Doutor, Universidade Federal do Rio Grande do Sul (UFRGS), Rua Washington Luiz, 855, Centro. CEP: 90010-460, Porto Alegre, RS. E-mail: ocgalli@ea.ufrgs.br

${ }^{3}$ Doutorando, Universidade Federal do Rio Grande do Sul (UFRGS), Rua Washington Luiz, 855, Centro. CEP: 90010 460, Porto Alegre, RS. UFRGS. E-mail: rfdecourt@ea.ufrgs.br
} 


\begin{abstract}
This work tests signaling theory in which investors receive payout announcement news from 1998 to 2006 regarding shares integrants of theoretical Index Ibovespa from January to April, 2006. To realize this research it was verified the presence of abnormal returns at the dates of payout announcements. Tests were made around the dates of announcement within a window of 11 days including the announcement date in an attempt to search for allowed information and to verify the feasibility and the arbitrage at this time window. These tests consider the presence of abnormal returns and accumulated returns when the market was operating in different levels of volatility and considering the magnitude of the dividend announced, trying to verify if the investors interpret and penalize the announcement of payout in symmetrical way regarding the magnitude and volatility variations. The results verifying presence of abnormal returns.
\end{abstract}

Keywords: Volatility; Payout Policy; The Signaling Theory; Dividends; Dividend Yield.

\title{
1 Introdução
}

A política de distribuição dos lucros ganhou terreno no campo científico com a publicação de Modigliani e Miller (1961), seguida do trabalho de Gordon (1963) e, especificamente, dos trabalhos sobre a teoria da sinalização de Pettit (1972), Bhattacharia (1979), Asquith e Mullins (1983), entre outros. Esses trabalhos procuravam identificar, resumidamente, os impactos que o pagamento dos proventos provocava no valor das ações.

Pode-se dizer que este impacto estava relacionado à condução de informação que os dividendos transmitiam aos investidores com relação ao futuro das empresas. Assim, o preço da ação oscilaria proporcionalmente ao pagamento dos dividendos. Logo, uma situação desejável em relação aos proventos seria satisfazer os acionistas com uma retenção de lucros para reinvestimento, seguida da distribuição de uma parcela otimizada dos recursos na forma de dividendos que maximizasse os ganhos dos acionistas.

Tendo como base o conceito da condução de informação, os pesquisadores publicaram uma série de estudos procurando variáveis de mercado que pudessem explicar o fenômeno da variação do valor das ações. Nesse sentido, uma das variáveis descobertas que podem afetar o valor das ações na distribuição dos lucros no Brasil foi a magnitude dos proventos distribuídos, conforme Procianoy e Verdi (2003), Freire e Lima (2003), Novis e Saito (2003), Kuronuna, Lucchesi e Fama (2004), entre outros, e que não pode ser comprovado nos trabalhos de Freire et al. (2005) e Firmino, Santos e Matsumoto (2002).

Além deste, outros fatores, como o efeito dos impostos sobre os proventos, o problema da assimetria de informações, a natureza dos contratos em andamento e dos custos de transação são variáveis que se relacionam diretamente com a política de distribuição dos lucros e influenciam o valor das ações, conforme Michaely e Allen (2002).

$\mathrm{Na}$ tentativa de encontrar outras variáveis que possam explicar este excesso de valorização dos ativos, esta pesquisa trata de examinar a obtenção de retornos anormais (retorno da ação menos o retorno do mercado) e retornos anormais acumulados conforme as variações de volatilidade do mercado e da magnitude do provento anunciado. Vale ressaltar que, no caso 
brasileiro, o termo provento pode ser considerado como pagamento de dividendos ou juros sobre capital próprio (JSCP), permitido pela Lei no. 9.249/95, art. 9.

A questão que deu origem à idéia para a realização desta pesquisa foi a suposição da presença de uma assimetria na variação dos retornos das ações com relação aos diferentes níveis de volatilidade em que o mercado estava operando, se com baixa, normal ou alta volatilidade, e conforme a magnitude do provento anunciado, se maior ou menor do que a média paga nos últimos anos, como fez Docking e Koch (2005). Este pressuposto, do modo como o mercado estaria operando, partiu de interpretações feitas de acordo com as variações do Ibovespa, na data do anúncio da distribuição dos proventos, pois este é o índice que representa as variações que aconteceram no mercado.

Assim, elaborou-se a seguinte pergunta: houve uma variação significativa na condução de informação conforme o nível de volatilidade em que o mercado estava operando na data do anúncio do pagamento dos proventos? Houve variação significativa na condução de informação conforme a magnitude do provento anunciado?

Dessa forma, este estudo se justifica como uma ferramenta de contribuição para que os administradores das empresas de capital aberto possam, de alguma forma, direcionar o pagamento dos proventos de acordo com as variações de volatilidade do mercado e de magnitude do provento que será pago, maximizando os benefícios para as firmas e para seus acionistas. Também se justifica como ferramenta de auxílio ao investidor marginal que procura investir nas melhores oportunidades que o mercado pode oferecer.

O objetivo principal deste trabalho foi o de verificar a assimetria de informações referente à percepção dos investidores com relação às tendências que o mercado apresentou e com relação à magnitud e do provento distribuído quando ocorreram anúncios do pagamento de proventos, procurando indícios de retornos anormais e retornos acumulados excessivos. Os cálculos foram realizados na data de anúncio do pagamento de dividendos, juros sobre capital próprio e pagamento concomitante. Esses cálculos também foram realizados numa janela de - 5 $\mathrm{a}+5$ dias ao redor das datas de anúncio.

\section{Fundamentação teórica}

A partir dos trabalhos de Lintner (1956) e Miller e Modigliani (1961) foi dado início a uma série de publicações que procuravam descrever e aprofundar os conhecimentos sobre a política de dividendos. Várias teorias surgiram como, por exemplo, a da irrelevância dos dividendos, de Miller e Modigliani (1961); a da relevância dos dividendos, de Gordon (1963); a do dividendo ótimo, de Masulis e Trueman (1988); e a teoria residual, entre outras.

A preocupação com a condução de informações e a relevância da distribuição dos lucros se tornou tema amplame nte explorado, surgindo o jargão de conhecimento comum que dizia que os investidores reagiam em sintonia ao pagamento dos dividendos.

A discussão da relevância e do conteúdo informacional foi complementada por Rees (1997), Strong (1997) e Brief e Zarowin (1999), sugerindo modelos para a precificação de ativos a partir de relações contábeis. Rees (1997) propôs um modelo de precificação em nível baseado no valor contábil, nos ganhos retidos, nos dividendos, no lucro retido, no investimento realizado e nas dívidas totais, semelhante aos utilizados por Ohlson (1995) e Strong, Walker e Harding (1996). 
Os resultados encontrados mostraram a relevância e o conteúdo informacional dos dividendos na valorização das firmas, num ambiente caracterizado pela assimetria de informação. Para Strong (1997), os resultados encontrados por Rees apenas poderiam ser interpretados como uma associação, e não, evidentemente, como uma sinalização ou relevância dos dividendos.

Seguindo na mesma linha de Rees (1997), Brief e Zarowin (1999) sugeriram a relevância dos dividendos e do conteúdo informacional por meio da estimação do valor das ações pelo valor contábil dos dividendos e dos ganhos auferidos. O trabalho de Brief e Zarowin foi inspirado e teve alguns resultados semelhantes aos obtidos nos papers de Ohlson (1995), Feltham e Ohlson (1995), Bernard (1995), Burgstahler e Dichev (1996), Collins, Maydew e Weiss (1997).

Outros trabalhos testaram diferentes variáveis para explicar a condução de informações, como o de Veronesi (1999) sobre a natureza das notícias - se boas ou ruins -, o de Baker, Veit e Powell (2001) sobre os fatores importantes para a determinação da política de dividendos tipo de indústria, dividendos passados, estrutura de capital, opinião dos executivos, etc. -, o de Mougoué e Rao (2003) sobre a relação entre dividendos pagos e a obtenção de resultados futuros, e o de Koch e Sun (2004) para os resultados passados (neste caso os dividendos deveriam ser interpretados como reações atrasadas das informações já recebidas pelos ganhos passados). Vale ressaltar que, no trabalho de Bernstein (1996), a teoria do conteúdo informacional não foi confirmada considerando a relação dividend yield e os retornos das ações.

Docking e Koch (2005) testaram a sensibilidade da reação do mercado no anúncio de mudanças no pagamento de dividendos no mercado americano. Os resultados mostraram retornos anormais significativos e positivos quando ocorreram anúncios da distribuição, com o mercado operando de forma normal ou em queda, mas com alta volatilidade, e quando ocorreram anúncios de diminuição de dividendos, com mercado em alta e com bastante volatilidade.

No Brasil, poucos estudos exploram a data de anúncio na política de dividendos, entre eles estão os trabalhos de Novis e Saito (2003), Agnes (2005) e Decourt, Procianoy e Pietro Neto (2007). O trabalho de Novis e Saito (2003) procurou analisar o preço das ações após o anúncio do pagamento dos dividendos, segmentando a amostra de acordo com o dividend yield (retorno sobre os dividendos). Os resultados encontrados revelaram a persistência dos retornos anormais para as empresas que pagaram as maiores relações dividend yield nos 90 dias analisados. Também foi constatado que as empresas que sofreram o processo de privatização apresentaram retornos anormais acumulados superiores às demais empresas da amostra.

O trabalho de Agnes (2005) analisou os anúncios de dividendos numa janela de 21 dias $(-10$ a +10$)$, com segmentações para dividendos, JSCP, pagamento simultâneo, ano do pagamento, setor, tipo de processo decisório e por tipo de controle acionário. Para a grande maioria das segmentações, a presença de retornos anormais foi próxima de zero e sem evidências estatísticas significativas para a amostra total. Outros resultados mostraram evidências de retornos anormais médios negativos para o pagamento dos JSCP e, para a segmentação por ano, apenas o ano 2000 apresentou mediana dos retornos de 2,59\%, significativa a 10\%.

Decourt, Procianoy e Pietro Neto (2007) testaram a teoria da sinalização por meio das alterações nos índices de payouts das empresas em comparação com a variação no lucro líquido do ano anterior e posterior em que ocorreu a distribuição dos proventos. Os principais resultados encontrados foram: as reduções no payout foram acompanhadas de diferenças significativas na variação do lucro líquido do exercício (que geralmente são conseqüência do aumento no lucro líquido do exercício) e as reduções no payout também sinalizaram crescimento do resultado futuro. 


\section{Método}

Para esta pesquisa, foi realizada uma análise quanto à presença de retornos anormais e retornos anormais acumulados, utilizando a metodologia de cálculo proposta por Mackinlay (1997), abaixo descrita, na busca de verificar a sinalização por parte dos investidores quanto ao impacto provocado pelo anúncio da distribuição dos lucros. Os cálculos foram realizados de maneira pontual, na data da divulgação das informações, e em uma janela de -5 a +5 dias em torno das datas de anúncio, procurando evidências de vazamentos de informações e da possibilidade de arbitragem em relação ao anúncio do pagamento dos proventos. O cálculo dos retornos anormais e acumulados foi realizado considerando-se níveis diferentes de volatilidade do mercado e em conformidade com a magnitude dos proventos anunciados.

A base de dados foi retirada do software Economática e do site da Bovespa com o preço de fechamento das ações ajustados pela inflação e pelos dividendos pagos. O período das datas de anúncio coletado está compreendido entre os anos de 1998 até 2006. O número de pagamento de proventos desse período foi de 845 eventos. Para evitar problemas de liquidez, esta análise foi realizada nas empresas que compõem a carteira teórica do Ibovespa de janeiro a abril de 2006.

\subsection{Cálculo dos retornos anormais}

Para calcular os retornos anormais, foi utilizada a diferença entre os retornos efetivos das ações e o retorno do mercado, representado pelo Ibovespa, obtido, algebricamente, pela equação:

$$
A R_{i t}=R_{i t}-R_{m t}
$$

em que $A R_{\text {it }}$ é o retorno anormal da ação i, na data t, e $R_{m t}$ é o retorno do mercado, calculado com base no Ibovespa, dado por:

$$
R_{m t}=\ln \left(\frac{\text { IBOV }_{t}}{\text { IBOV }_{t-1}}\right)
$$

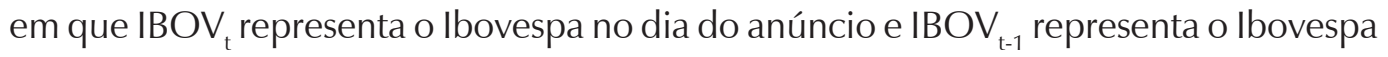
na data anterior à data do anúncio da distribuição dos proventos. O retorno das ações foi calculado da mesma maneira que os retornos do mercado.

Também foi realizado o cálculo dos retornos anormais acumulados, conforme Mackinlay (1997), estimados pelo valor dos retornos anormais de um período adicionado aos retornos anormais dos períodos subseqüentes, calculados num intervalo de 11 dias, ou seja, no período de -5 a +5 em torno da data do anúncio e que foram obtidos da seguinte maneira:

$$
\operatorname{CAR}_{(\mathrm{t} 1, \mathrm{t} 2)}=\sum_{\mathrm{t}=\mathrm{t} 1}^{\mathrm{t} 2} \mathrm{AR}_{\mathrm{it}}
$$

em que $\mathrm{CAR}_{(t 1, t 2)}$ é o retorno anormal acumulado para a ação i do tempo $t_{1}$ até o tempo $\mathrm{t}_{2}$. 
Para o cálculo dos retornos anormais médios, foi utilizada a seguinte equação:

$$
\overline{\mathrm{AR}_{\mathrm{t}}}=\frac{1}{\mathrm{~N}} \sum_{\mathrm{i}=1}^{\mathrm{N}} \overline{\mathrm{AR}_{\mathrm{it}}}
$$

em que $\overline{\mathrm{AR}}$ é a média dos retornos anormais. Essa fórmula também foi aplicada para o período ao redor da data do anúncio. Esse teste foi realizado para verificar se os retornos anormais persistem após a realização da data do evento e se houve "vazamento de informações" nos períodos anteriores à convocação para a realização das assembléias.

\subsection{O cálculo da magnitude dos proventos}

Para calcular a magnitude dos dividendos, foi utilizada a fórmula:

$$
\mathrm{DY}=\frac{\mathrm{D}}{\mathrm{P}_{\mathrm{t}-1}}
$$

em que DY é o dividend yield, $\mathrm{D}$ é o montante de dividendos pagos por ação e $\mathrm{P}_{\mathrm{t}-1}$ é o preço da ação no dia anterior ao da data do anúncio. Esse cálculo foi utilizado como referência para a subdivisão dos dividendos em duas categorias: a de proventos maiores e a de proventos menores.

A categoria de proventos menores foi composta pelos proventos pagos que se encontraram abaixo da média histórica paga para todas as ações da amostra, até os dividendos que se encontraram na média, inclusive. A categoria dos proventos maiores foi composta pelos proventos pagos que se situaram acima da média histórica dos proventos pagos.

\subsection{Padronização da volatilidade}

Três níveis de volatilidade foram estabelecidos, denominados de alta, normal e baixa volatilidade. Para a determinação de mercado com alta volatilidade, normal ou baixa volatilidade, também foi utilizada a fórmula (2) e se assumirá, inicialmente, que os retornos do Ibovespa sejam normais. Logo, como critério subjetivo, foi adotado que, após a padronização dos retornos, estando o retorno do Ibovespa situado entre a média e 0,3 desvios padrões para a direita ou para a esquerda, o mercado estava operando com baixa volatilidade. Caso estivesse operando entre 0,3 e 0,85 desvios padrões em relação à média estaria operando de forma normal, e acima de 0,85 desvios padrões o mercado estaria operando com alta volatilidade.

Esta divisão foi realizada para que a amostra ficasse segmentada de maneira a ter aproximadamente $33 \%$ da amostra para cada subdivisão considerando a volatilidade. Graficamente, ter-se-ia a seguinte situação para a segregação em alta, baixa e volatilidade normal: 


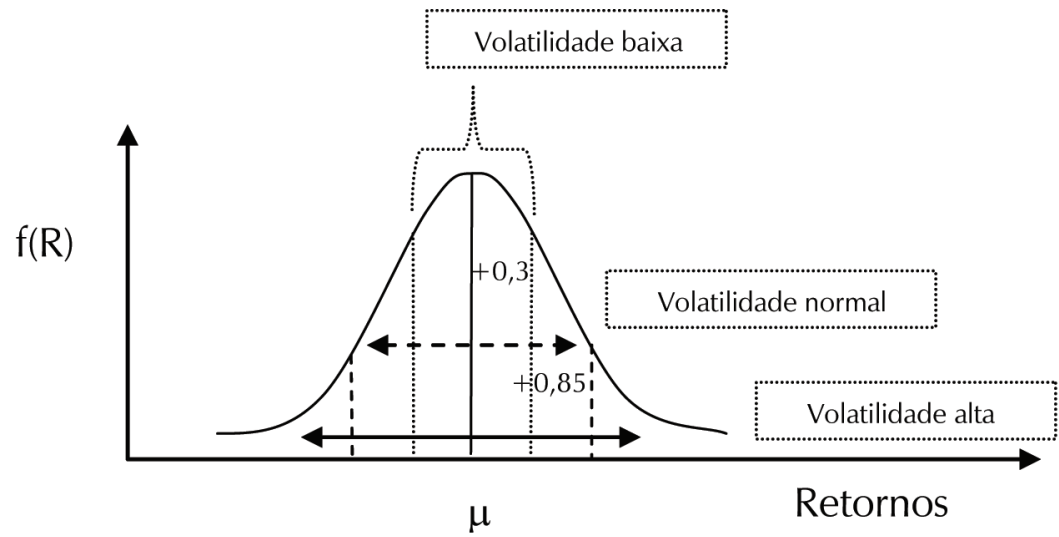

Figura 1 - Curva normal e segmentação por volatilidade.

\subsection{A amostra}

A amostra foi composta pelas datas dos anúncios do pagamento dos proventos das empresas componentes do índice Bovespa, carteira teórica referente aos meses de janeiro a abril de 2006, perfazendo um total de 57 empresas. Na Tabela 1, relacionam-se as empresas componentes da amostra. Dessas 57 empresas, a Contax e a Net foram excluídas por não apresentarem pagamento de proventos ou dados dos preços das ações; dessa forma, a amostra final foi de 55 empresas. Também foram excluídas as datas de anúncio que apresentaram um valor de 3,5 desvios padrões para mais ou para menos da média dos retornos obtidos, por serem consideradas observações atípicas e para melhorarem a veracidade dos resultados obtidos, conforme Hair et al. (2005).

Foram consideradas datas de anúncios do pagamento dos proventos aquelas em que a notícia foi publicada pela primeira vez no site da empresa, jornais ou no site da Bovespa, referentes às datas da realização das Assembléias Gerais Ordinárias (AGO) ou das Reuniões do Conselho de Administração (RCA).

Tabela 1 - Empresas componentes da amostra utilizada para a pesquisa

\begin{tabular}{|l|l|l|l|l|l|}
\hline Código & Ação & Tipo & Código & Ação & Tipo \\
\hline ACES4 & ACESITA & PN EJ & ITAU4 & ITAUBANCO & PN EJ \\
\hline AMBV4 & AMBEV & PN EDJ & ITSA4 & ITAUSA & PN EJ \\
\hline ARCZ6 & ARACRUZ & PNB EJ & KLBN4 & KLABIN S/A & PN \\
\hline ARCE3 & ARCELOR BR & ON & LIGH3 & LIGHT & ON \\
\hline BBDC4 & BRADESCO & PN & PETR3 & PETROBRAS & ON EJ \\
\hline BRAP4 & BRADESPAR & PN & PETR4 & PETROBRAS & PN EJ \\
\hline BBAS3 & BRASIL & ON & SBSP3 & SABESP & ON *EJ \\
\hline BRTP3 & BRASIL T PAR & ON & SDIA4 & SADIA S/A & PN EJ \\
\hline BRTP4 & BRASIL T PAR & PN & CSNA3 & SID NACIONAL & ON \\
\hline BRTO4 & BRASIL TELEC & PN & CRUZ3 & SOUZA CRUZ & ON EJ \\
\hline BRKM5 & BRASKEM & PNA EJ & TCOC4 & TELE CTR OES & PN \\
\hline CMET4 & CAEMI & PN & TLCP4 & TELE LEST CL & PN \\
\hline CLSC6 & CELESC & PNB EJ & TNLP3 & TELEMAR & ON \\
\hline CMIG3 & CEMIG & ON & TNLP4 & TELEMAR & PN \\
\hline CMIG4 & CEMIG & PN & TMAR5 & TELEMAR N L & PNA \\
\hline
\end{tabular}

Rev. Adm. UFSM, Santa Maria, v. I, N. 1, P. 37-56, JAN./ABr. 2008 
VOLATILIDADE, MAGNITUDE DOS PROVENTOS E A SINALIZAÇÃO...

(continuação da Tabela 1)

\begin{tabular}{|l|l|l|l|l|l|}
\hline Código & Ação & Tipo & Código & Ação & Tipo \\
\hline CESP4 & CESP & PN & TMCP4 & TELEMIG PART & PN \\
\hline CGAS5 & COMGAS & PNA & TLPP4 & TELESP & PN EJ \\
\hline CPLE6 & COPEL & PNB & TSPP4 & TELESP CL PA & PN \\
\hline CRTP5 & CRT CELULAR & PNA & TCSL3 & TIM PART S/A & ON \\
\hline ELET3 & ELETROBRAS & ON & TCSL4 & TIM PART S/A & PN \\
\hline ELET6 & ELETROBRAS & PNB & TRPL4 & TRAN PAULIST & PN EJ \\
\hline ELPL4 & ELETROPAULO & PN & UBBR11 & UNIBANCO & UNT EJ \\
\hline EMBR3 & EMBRAER & ON EJ & USIM5 & USIMINAS & PNA EJ \\
\hline EMBR4 & EMBRAER & PN EJ & VCPA4 & V C P & PN EJ \\
\hline EBTP4 & EMBRATEL PAR & PN & VALE3 & VALE R DOCE & ON \\
\hline GGBR4 & GERDAU & PN & VALE5 & VALE R DOCE & PNA \\
\hline GOAU4 & GERDAU MET & PN & & & \\
\hline PTIP4 & IPIRANGA PET & PN & & & \\
\hline
\end{tabular}

Fonte: Site Bovespa.

Os cálculos foram realizados para a amostra total sem segmentação por setor de atuação sendo que oito datas de anúncio de distribuição de JSCP foram automaticamente excluídas por falta de liquidez e uma referente ao anúncio de distribuição de dividendos. Dessa forma, a amostra ficou composta de 837 observações, sendo 148 observações para o pagamento de dividendos, 569 para a distribuição de JSCP e 54 para distribuiç̧ão de juros e dividendos simultaneamente.

\subsection{As hipóteses testadas}

Para efetivar a realização deste trabalho, foram testadas as seguintes hipóteses quanto à presença de retornos anormais:

- H0: não há retornos anormais na janela de estudo do evento;

- H1: Há retornos anormais na janela de estudo do evento.

Também foram testadas as seguintes hipóteses quanto à simetria dos retornos:

- H0: os retornos anormais não são simétricos em relação à volatilidade e à magnitude do provento pago;

- H1: os retornos anormais são simétricos em relação à volatilidade e à magnitude do provento pago.

\section{Os resultados}

\subsection{Retornos anormais médios e acumulados - volatilidade}

Para a subamostra de volatilidade baixa, os resultados para os retornos anormais médios foram de 0,198 e de 57,53\% para os retornos anormais acumulados, com 287 observações efetivas entre pagamento de juros sobre capital próprio e dividendos. Esses resultados mostraram-se maiores em relação aos obtidos quando o mercado operava com volatilidade normal, apresentados a seguir.

Para os anúncios realizados com o mercado operando com volatilidade normal, os retornos anormais médios encontrados foram de 0,195 e de 59,33\% para os retornos anormais acumulados, com 304 datas de anúncios. Esses resultados contrariam as hipóteses esperadas de 
que, com o aumento da volatilidade do mercado, os investidores assumiriam maiores riscos e, portanto, obteriam maiores retornos em seus investimentos.

$\mathrm{Na}$ análise dos retornos anormais acumulados, foi evidenciado que os resultados apontam para uma deficiência no mercado, pois se esperava que a presença de retornos anormais acumulados aumentasse com o aumento da volatilidade do mercado. No entanto, os retornos anormais médios foram inferiores, e o resultado obtido se deve ao maior número de datas de anúncio.

Para a subamostra de retornos com alta volatilidade, os resultados foram bastante altos em relação às duas outras subamostras. Os retornos anormais médios encontrados foram de 0,345 contra 0,195\%, para volatilidade normal, e 0,198\% para volatilidade baixa.

A possibilidade da obtenção de retornos anormais acumulados foi a maior registrada, com 84,93\%, com 246 observações, e este resultado mostrou-se compatível com a hipótese do risco/retorno do investidor com relação a excessos de volatilidade no mercado, pois é de se esperar que mercados muito voláteis produzam retornos excessivos maiores, portanto, possibilidades de obtenção de lucros também maiores devido ao aumento do risco de se investir.

A Figura 2 apresenta o quadro resumo para facilitar a comparação dos retornos anormais acumulados, dividindo-se a amostra total de acordo com a volatilidade do mercado. Pela análise da Figura 2, percebe-se que há uma pequena diferença nos retornos quando o mercado estava operando em baixa e de maneira normal, conforme os critérios estabelecidos, e, quando o mercado esta operando com alta volatilidade, fica evidente a possibilidade de obtenção de retornos excessivos maiores neste tipo de operação pontual.

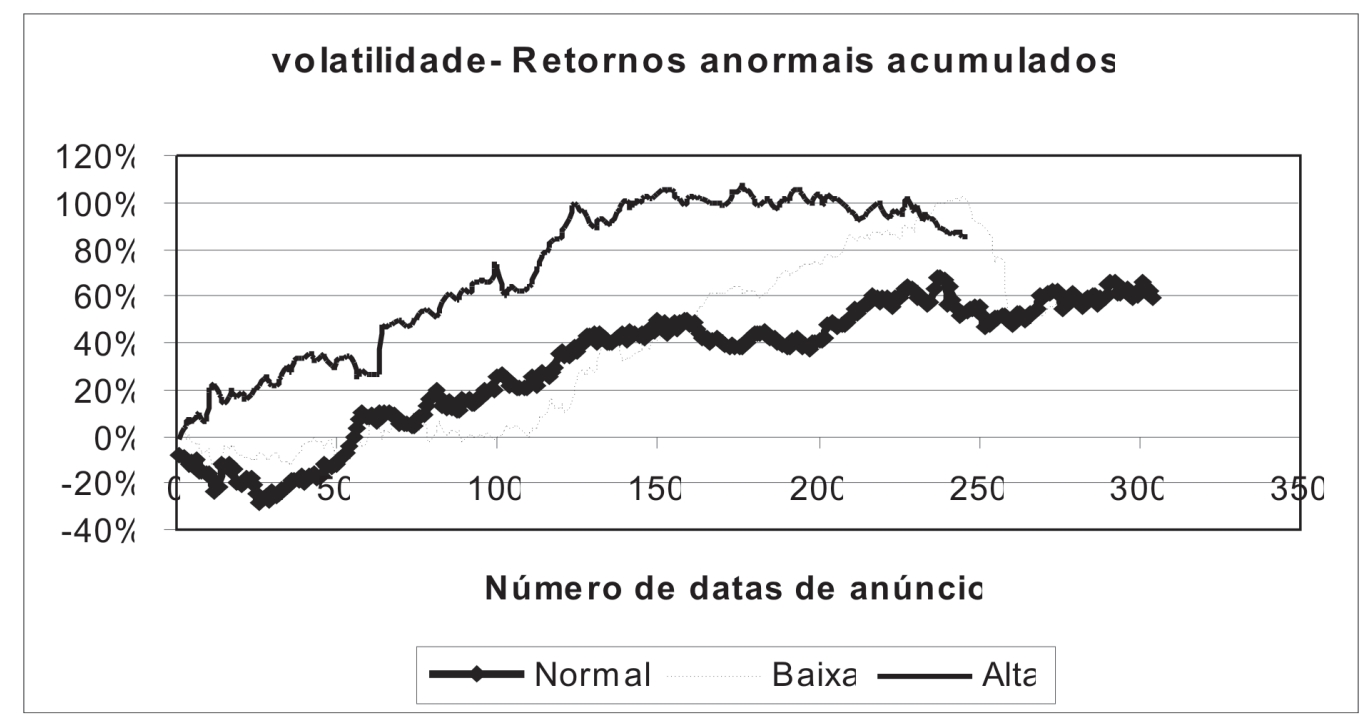

Figura 2 - Quadro resumo de retornos anormais acumulados conforme variação da volatilidade no mercado.

\subsection{Retornos anormais médios e acumulados - de -5 a +5 - volatilidade}

Para a subamostra de baixa volatilidade no período de -5 a +5 dias, os retornos anormais médios encontrados foram da ordem de 0,196\%, próximo do valor encontrado para os retornos obtidos para as observações nas datas de anúncio com volatilidade baixa e normal. 
$\mathrm{Na}$ análise dos retornos anormais acumulados, o resultado encontrado foi de $607,65 \%$, resultado contrastante se comparado com a subamostra dividida quando o mercado estava operando de maneira normal e com alta volatilidade, pois era de se esperar que os retornos acumulados nessa subamostra possuísse o menor percentual acumulado para retornos excessivos. Verificou-se, nessa subamostra, a presença de 287 datas de anúncio e seus respectivos dias ao redor delas.

Para a subamostra com o mercado operando com volatilidade normal, obteve-se 304 datas de anúncio, e os retornos anormais médios encontrados para os dias ao redor dessas datas de divulgação foram de $0,121 \%$. Esse resultado oferece números contraditórios, tendo em vista que era de se esperar que os resultados sugerissem retornos excessivos maiores devido ao aumento no risco dos investimentos. Já, para os retornos anormais acumulados, os resultados obtidos foram de $400,35 \%$.

Para a subamostra com alta volatilidade, os retornos anormais médios encontrados foram de $0,094 \%$. Esse resultado também é contraditório do ponto de vista do risco/retorno assumido no mercado financeiro, confirmando, mais uma vez, a contradição na relação esperada para retornos excessivos. O número de datas de anúncio para esta modalidade foi de 246 datas.

Os resultados encontrados para os retornos anormais acumulados foram de 249,31\%, inferiores aos resultados encontrados para a subamostra com baixa volatilidade e com volatilidade normal. Esses resultados também se contradizem em relação ao paradigma do risco e retorno, no qual o investidor racional busca retornos maiores quanto maior o risco assumido.

Para as duas modalidades de retornos calculados - anormais e anormais acumulados -, os resultados foram contraditórios em relação aos esperados anteriormente à realização dos testes empíricos, o que sugere a obtenção de retornos excessivos seguindo as tendências do paradigma de risco e retorno. Como se observa na Figura 3, os maiores retornos excessivos acumulados foram encontrados quando o mercado estava operando em baixa volatilidade. Uma possibilidade para os resultados encontrados divergirem tanto dos esperados poderia ser o tamanho da amostra utilizada para cada subamostra, no entanto, as subamostras tiveram número de observações semelhantes.

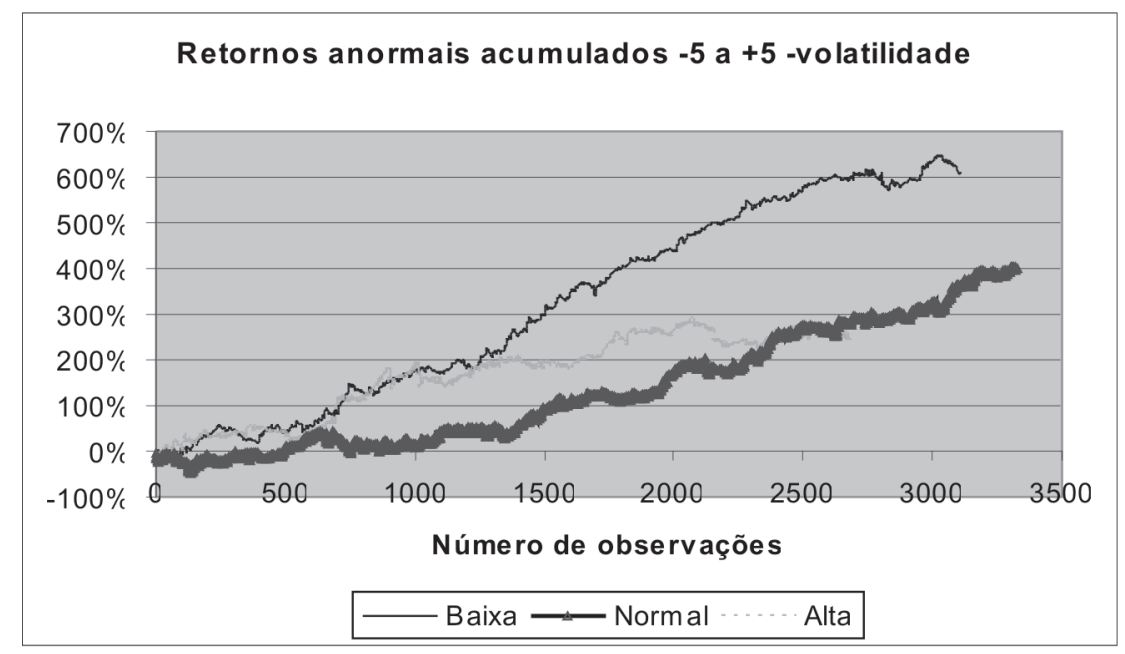

Figura 3 - Quadro resumo de retornos anormais acumulados entre -5 e +5 conforme volatilidade de mercado. 
Para aprofundar os conhecimentos a respeito dos retornos anormais, confeccionouse um gráfico para a média dos retornos anormais médios na janela de evento em estudo, conforme a Figura 4.

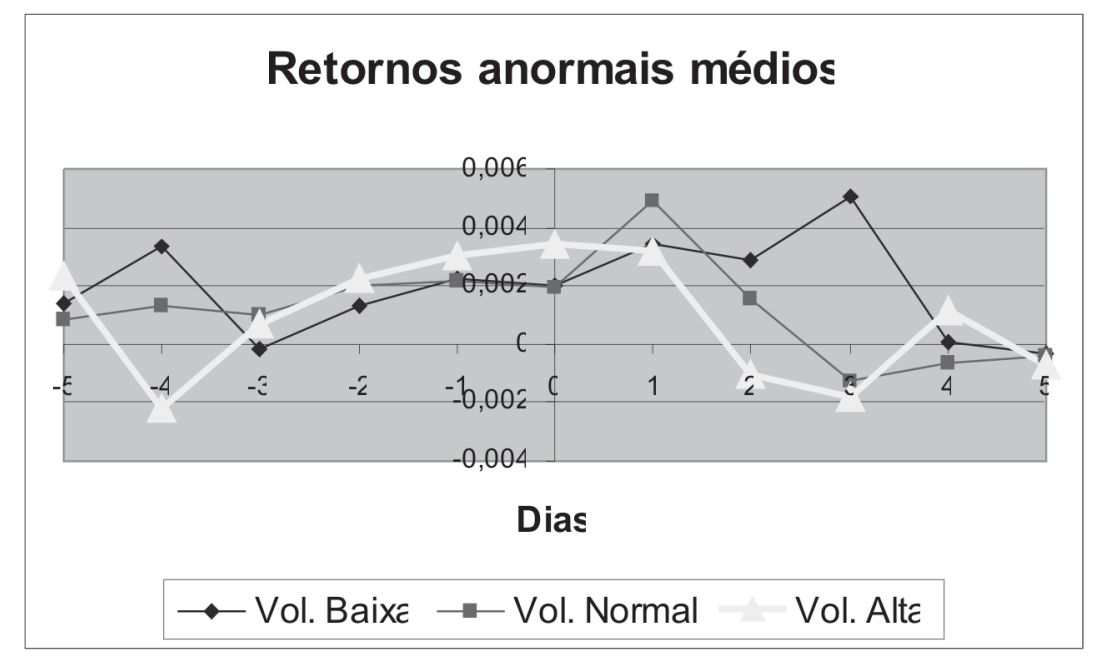

Figura 4 - Retornos anormais médios - volatilidade - de -5 a +5 .

Uma das conclusões que se pode extrair do gráfico apresentado na Figura 4 é que, para a volatilidade baixa, os retornos anormais médios são mais constantes; já, para a volatilidade alta, pode-se notar que há uma variação mais acentuada na janela em estudo.

\subsection{Retornos anormais médios e acumulados - magnitude}

Dividindo-se a amostra e realizando-se o teste em relação à magnitude do dividendo anunciado, os resultados encontrados, para os dividendos maiores do que as médias pagas, apresentaram retornos anormais médios de 0,292\%. Esse resultado evidencia que, na média, os proventos com magnitudes maiores do que a média paga no mercado oferecem uma possibilidade de arbitragem para a obtenção de retornos anormais médios maiores do que em relação aos dividendos menores.

Para a análise de retornos acumulados, os resultados obtidos foram de 78,56\% inferiores aos resultados encontrados para os retornos acumulados quando o dividendo pago era menor do que a média. O número de datas de anúncio analisadas foi de 269 dias.

Para a segmentação da amostra, considerando-se os proventos pagos menores do que a média, os resultados encontrados para os retornos anormais foram de 0,2025\% para uma subamostra de 477 datas de anúncio. Esses resultados confirmam trabalhos anteriormente publicados em relação à magnitude dos proventos pagos.

Já, para os retornos anormais acumulados, os resultados encontrados foram de 116,84\% superiores aos obtidos com os pagamentos de maior magnitude, no entanto, salienta-se que o número de observações que contribuíram para a formação deste resultado é muito superior ao número de observações analisadas para a amostra de dividend yield maior.

Para facilitar as comparações de acordo com as segmentações proposta, elaborou-se um quadro resumo das duas subdivisões, apresentado na Figura 5. 


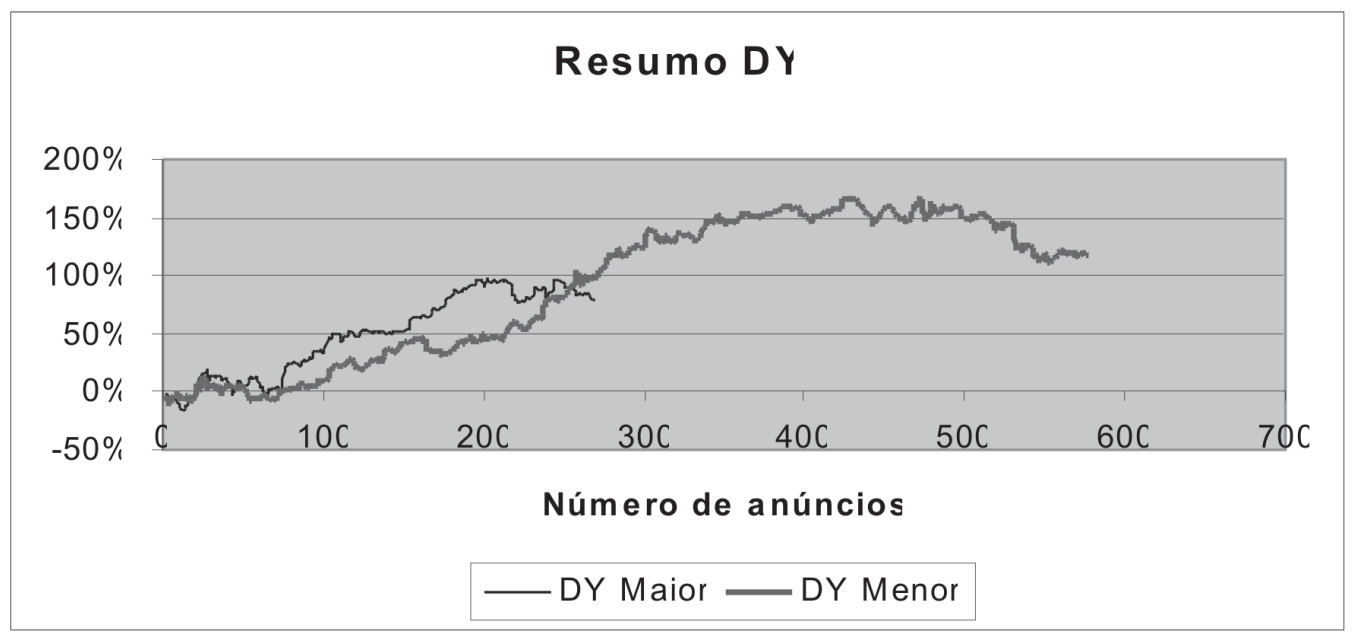

Figura 5 - Resumo DY de $-5 \mathrm{a}+5$.

Ainda no intuito de aprofundar os conhecimentos da amostra analisada, elaborou-se um quadro comparativo para a janela de eventos em análise, mostrando os retornos anormais médios, conforme Figura 6.

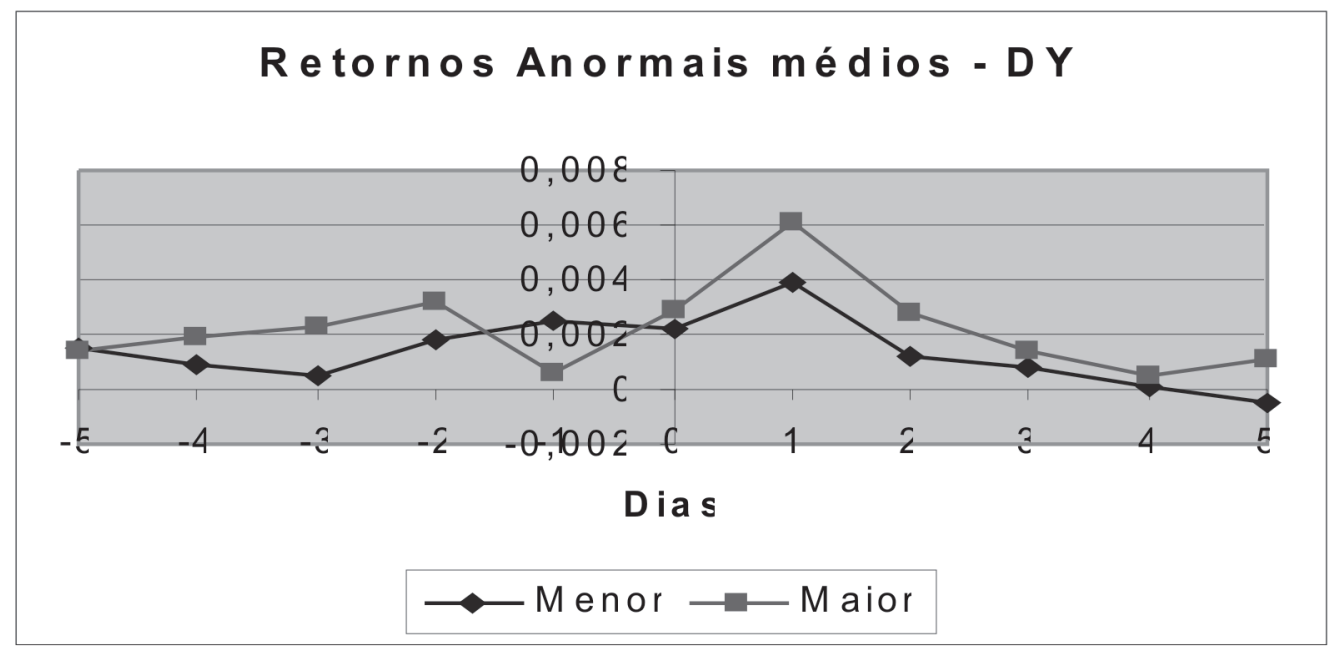

Figura 6 - Retornos anormais médios DY de -5 a +5 .

A Figura 6 evidencia que os retornos anormais médios são maiores para a subamostra com DY maior e que no dia 1 após a data de anúncio ocorre um significativo aumento dos retornos anormais médios.

Para verificar se os resultados encontrados são significativos entre os dias da janela em estudo, aplicou-se um teste de diferença de médias do tipo ANOVA - Análise de Variância (one way ANOVA) - dentro da amostra total para cada grupo de mesmo dia, utilizando o software SPSS. A hipótese nula, neste caso, é a de que a variância dos retornos entre os grupos de dias (cinco dias antes da data de anúncio, quatro dias antes da data de anúncio etc.) é igual para todos os dias, ou seja, que ela não é estatisticamente diferente entre os grupos. Esse teste é necessário porque presume uma das condições necessárias para a realização do teste $t$ de Student, que foi utilizado para verificar se os retornos são diferentes de zero, pois possuem uma média muito próxima desse valor. 
Como se pode observar dos resultados apresentados na Tabelas 2, não se pode aceitar a hipótese nula de que a variância é igual para todos os dias. Conforme valor da estatística $\mathrm{F}$ de Snedecor, há um nível de significância de 5\%, pois este valor ultrapassa o valor crítico de 1.8307 para a amostra com 10 graus de liberdade para a variância amostral das médias entre os 11 dias da janela em estudo e infinitos graus de liberdades para a variância amostral de cada grupo de dias.

Tabela 2 - ANOVA

\begin{tabular}{|l|r|r|r|r|r|}
\hline & $\begin{array}{r}\text { Sum of } \\
\text { Squares }\end{array}$ & \multicolumn{1}{|c|}{ Df } & $\begin{array}{c}\text { Mean } \\
\text { Square }\end{array}$ & F & Sig. \\
\hline $\begin{array}{l}\text { Between } \\
\text { Groups }\end{array}$ &, 013 & 10 &, 001 & 2,347 &, 009 \\
\hline $\begin{array}{l}\text { Within } \\
\text { Groups }\end{array}$ & 4,869 & 9125 &, 001 & & \\
\hline Total & 4,882 & 9135 & & & \\
\hline
\end{tabular}

Para visualizar a variabilidade nas médias dos retornos para cada dia, elaborou-se o gráfico apresentado na Figura 7. Dessa forma, a visualização das diferenças entre as médias dos retornos torna-se evidente. Percebe-se uma diferença grande em relação ao dia 1 após as datas dos anúncios e os dias 4 e 5 após as datas dos anúncios.

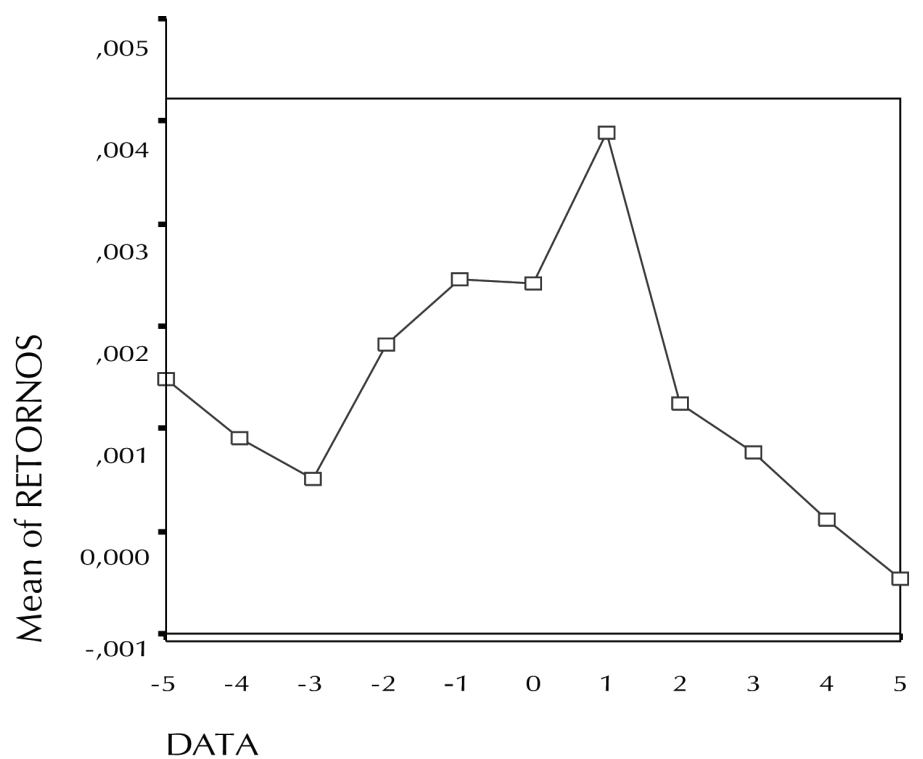

Figura 7 - Gráfico de diferença de médias.

Tendo em vista os resultados encontrados, do gráfico de médias e da diferença de variância para os dias analisados, torna-se necessária uma análise a posteriori, para verificar a homogeneidade da variância e confirmar o resultado global obtido pela estatística F, comparando a possibilidade de haver diferenças na variância entre os dias específicos.

Para isso, aplicou-se um teste de diferença de médias para a variância do tipo ANOVA para múltiplas comparações e seguindo a metodologia de Tukey HSD. Os resultados são mostrados na Tabela 3, em que a variância da média dos retornos para os dias 1, 4 e 5 são 
significativamente diferentes, ou seja, há um excesso de variabilidade nos retornos anormais para essas datas específicas ao redor da data de anúncio. Esses resultados confirmam as estatísticas obtidas com relação ao teste $\mathrm{F}$ e especificam em que dias ocorre a diferença de variância na amostra analisada.

Tabela 3 - Teste de diferença de médias de Tukey HSD de -5 a +5 .

\begin{tabular}{|l|l|r|r|r|r|r|}
\hline (I) data & (J) data & Mean difference (I-J) & Std. Error & Sig. & \multicolumn{2}{|c|}{$95 \%$ Confidence Interval } \\
\hline & & & & & Lower bound & Upper Bound \\
\hline & 1 &,- 00147629 &, 001131928 &, 968 &,- 00512050 &, 00216793 \\
\hline & 2 &, 00116940 &, 001132960 &, 995 &,- 00247814 &, 00481694 \\
\hline & 3 &, 00163488 &, 001132271 &, 937 &,- 00201044 &, 00528021 \\
\hline & 4 &, 00229663 &, 001132271 &, 629 &,- 00134870 &, 00594195 \\
\hline & 5 &, 00287488 &, 001130222 &, 279 &,- 00076384 &, 00651360 \\
\hline 1 & -5 &, 00239163 &, 001132602 &, 569 &,- 00125475 &, 00603802 \\
\hline & -4 &, 00297815 &, 001134642 &, 236 &,- 00067481 &, 00663110 \\
\hline & -3 &, 00337417 &, 001134985 &, 101 &,- 00027989 &, 00702823 \\
\hline & -2 &, 00206328 &, 001134300 &, 769 &,- 00158858 &, 00571513 \\
\hline & -1 &, 00143277 &, 001133619 &, 975 &,- 00221689 &, 00508243 \\
\hline & 0 &, 00147629 &, 001131928 &, 968 &,- 00216793 &, 00512050 \\
\hline & 2 &, 00264568 &, 001135672 &, 413 &,- 00101059 &, 00630195 \\
\hline & 3 &, 00311117 &, 001134985 &, 182 &,- 00054289 &, 00676523 \\
\hline & 4 &, $00377291\left(^{*}\right)$ &, 001134985 &, 036 &, 00011885 &, 00742697 \\
\hline & 5 &, $00435117\left(^{*}\right)$ &, 001132940 &, 006 &, 00070369 &, 00799864 \\
\hline
\end{tabular}

* The mean difference is significant at the .05 level

A Tabela 3 faz múltiplas comparações entre os dias da janela do evento em estudo, mostrando que somente nos dias 1, 4 e 5 não se pode assumir, ao nível de 5\% de significância, que as variâncias dos retornos médios entre os dias não são estatisticamente iguais.

A partir dessas análises é conveniente ressaltar que, como a maioria das séries financeiras não assume distribuição normal, pode surgir um problema nos resultados obtidos pelas estatísticas que assumem a normalidade das séries e que provocam, portanto, diferenças no valor do teste $\mathrm{F}$. Nesse sentido, aplicou-se um teste para verificar a normalidade da série dos retornos anormais de -5 a +5 , conforme descrito a seguir.

Para testar a normalidade da série dos retornos anormais, utilizou-se o teste de Jarque-Bera, com o software Eviews 4.1, conforme Figura 8.

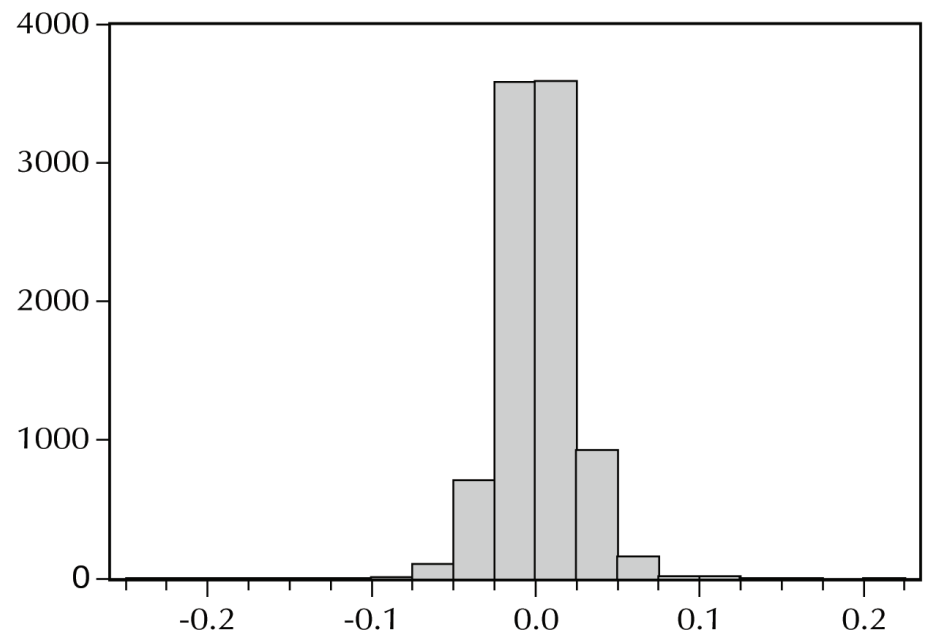

\begin{tabular}{|lr|}
\hline \multicolumn{2}{|l|}{ Series: RA } \\
Sample 19209 \\
\multicolumn{2}{|l|}{ Observations 9138} \\
Mean & 0.001375 \\
Median & 0.000740 \\
Maximum & 0.210704 \\
Minimum & -0.228007 \\
Std. Dev. & 0.023116 \\
Skewness & -0.022855 \\
Kurtosis & 8.892063 \\
& \\
Jarque-Bera & 13219.07 \\
Probability & 0.000000 \\
\hline
\end{tabular}

Figura 8 - Histograma retornos anormais.

Rev. Adm. UFSM, SAnta Maria, v. I, N. 1, P. 37-56, JAN./ABr. 2008 
Os resultados mostram que a série analisada não pode ser considerada normal, pois a estatística de Jarque-Bera é muito superior ao seu limite crítico de três unidades. Pela análise da Figura 8, podemos notar que além de não ser normal, a série dos retornos obtidos é assimétrica à esquerda, conforme resultados do coeficiente de Skewness.

Com esses resultados, pode-se levantar a hipótese de que haja ambigüidade estatística na análise da variância proveniente da não normalidade das séries utilizadas, no entanto, conforme Boneau (1960), a utilização do teste t e $F$ para séries de amostragens grandes são robustas e confiáveis, mesmo no caso da violação das premissas necessárias (homogeneidade de variâncias, normalidade e simetria dos dados), sugerindo que os resultados dessas anormalidades provocam efeitos mínimos nos resultados dessas estatísticas e que são atenuados pelo tamanho da amostra.

Para resolver essa aparente contradição levantada, foi utilizado o teste para comparação de médias de Wilcoxon, não paramétrico, sugerido por Brown e Warner (1980) e MacKinlay (1997), para que os resultados escolhidos sejam robustos e que validem os resultados obtidos pelos testes paramétricos. A hipótese nula, neste caso, é de que não há diferenças entre a variância dos grupos analisados.

Essa comparação foi realizada na tentativa de encontrar diferenças significativas entre os dias corridos, ou seja, entre os dias -5 e -4, -4 e -3... 4 e 5.

Como se pode observar da Tabela 4, em nenhum dos dias da janela de evento em estudos o valor de Z ultrapassa seus valores críticos, ao nível de significância de 5\%, de 1,96. Portanto, apesar de os testes paramétricos terem confirmado presença de diferenças estatísticas na variância média dos retornos entre os dias 1, 4 e 5, os resultados dos testes não-paramétricos rejeitam a hipótese de que haja diferença entre a variância dos retornos da janela em estudos.

Tabela 4 - Valor de Z para o Teste Wilcoxon 5\%

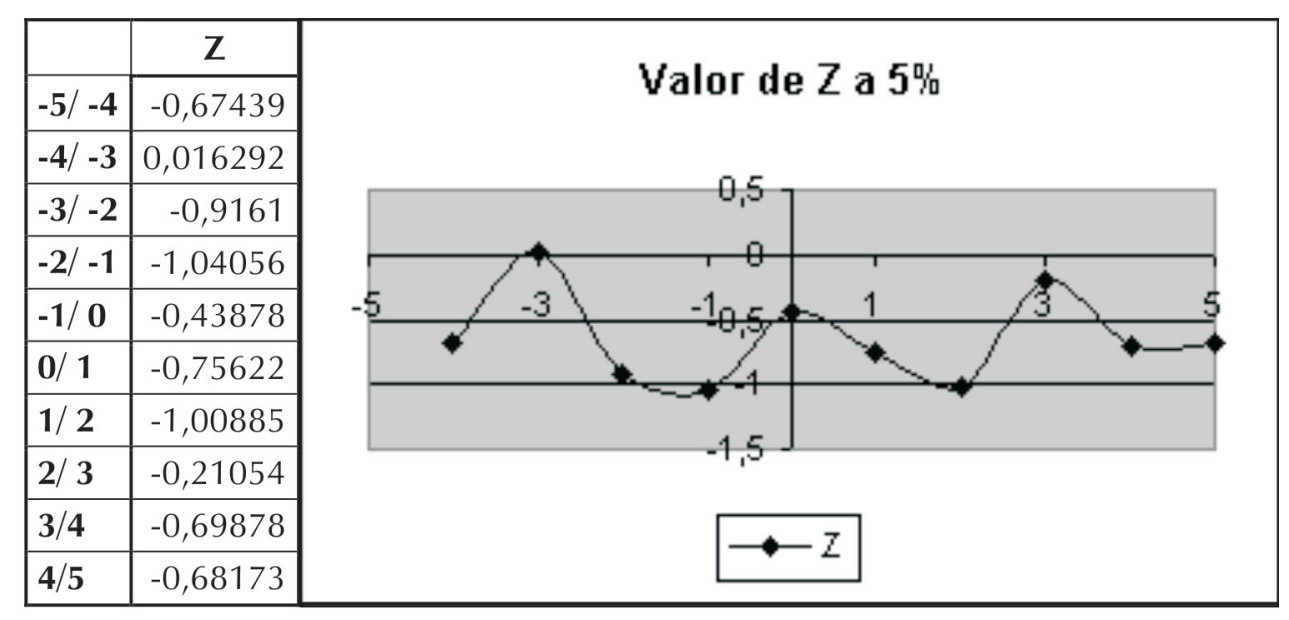

Deve-se considerar, no entanto, que o teste de igualdade de variâncias de Tukey HSD foi realizado por múltiplas comparações. Já, para o teste de Wilcoxon, somente para os dias seguidos e que o teste de sinais de Wilcoxon pode ter problemas de especificação quando a distribuição analisada possui problemas de assimetria, conforme Mackinlay (1997). Dessa forma, optou-se pela realização de outro teste não-paramétrico por postos na tentativa de resolver a ambigüidade estatística encontrada. O teste escolhido foi o de Kruskal-Wallis, e os resultados são mostrados na Tabela 5 . 
Tabela 5 - Teste de Kruskall-Wallis

\begin{tabular}{|l|l|r|r|}
\hline & DATA & \multicolumn{1}{|c|}{ N } & Mean Rank \\
\hline RETORNOS & -5 & 837 & 4613,73 \\
\hline & -4 & 837 & 4548,94 \\
\hline & -3 & 837 & 4538,48 \\
\hline & -2 & 837 & 4680,86 \\
\hline & -1 & 837 & 4664,59 \\
\hline & 0 & 837 & 4762,04 \\
\hline & 1 & 837 & 4873,73 \\
\hline & 2 & 837 & 4546,36 \\
\hline & 3 & 837 & 4585,79 \\
\hline & 4 & 837 & 4472,99 \\
\hline & 5 & 837 & 4356,49 \\
\hline & Total & 9207 & \\
\hline
\end{tabular}

Como se pode ver na Tabela 5, é evidenciado o nivelamento dos dados analisados entre os dias com o estabelecimento de um posto médio para cada data da janela em estudo. A partir desses dados, é feita a comparação através de um teste qui-quadrado para verificar se a variância entre os dias é semelhante. A hipótese nula, nesse caso, é a de que a variância é igual para todos os dias. Os resultados são mostrados na Tabela 6.

Tabela 6 - Estatísticas do teste de Kruskal-Wallis (a, b)

\begin{tabular}{|l|r|}
\hline & RETORNOS \\
\hline Chi-Square & 23,317 \\
\hline df & 10 \\
\hline Asymp. Sig. &, 010 \\
\hline
\end{tabular}
a Kruskal Wallis Test
b Grouping Variable: DATA

Como se pode notar, a estatística qui-quadrado ultrapassa seu valor crítico de 18,30, sugerindo que a amostra analisada não possui variância igual para todos os dias da janela em estudo. Esses resultados confirmam os obtidos pelos testes paramétricos e contrariam os resultados do teste de Wilcoxon; logo, os resultados adotados foram os de que a amostra analisada possui diferença para a variância entre os dias da janela em estudo, resultados que podem levar a diferenças mínimas na análise do teste t, conforme Boneau (1960).

Para melhorar o conhecimento dos dados analisados, é necessário saber se, além dos resultados, em termos de variância, serem estatisticamente iguais, a média dos retornos pode ser considerada estatisticamente diferente de zero. Para checar essa hipótese, aplicou-se um teste do tipo t de Student, considerando a hipótese nula de que os retornos de cada dia $(-5,-4 \ldots)$ são estatisticamente iguais a zero, o nível de significância utilizado foi de $1 \%$, obtendose os dados da Tabela 7. 
Tabela 7 - Teste t para média dos retornos diferentes de 0

\begin{tabular}{|c|c|c|c|c|c|c|c|}
\hline \multirow[t]{3}{*}{ DATA } & & \multicolumn{6}{|c|}{ Test Value $=0$} \\
\hline & & \multirow[t]{2}{*}{$T$} & \multirow[t]{2}{*}{ df } & \multirow[t]{2}{*}{$\begin{array}{l}\text { Sig. }(2- \\
\text { tailed) }\end{array}$} & \multirow[t]{2}{*}{$\begin{array}{l}\text { Mean } \\
\text { Difference }\end{array}$} & \multicolumn{2}{|c|}{$\begin{array}{l}\text { 99\% Confidence Interval of the } \\
\text { Difference }\end{array}$} \\
\hline & & & & & & Lower & Upper \\
\hline-5 & RETORNOS & 1,888 & 834 &, 059 & 00149265 &,- 00054829 & 00353360 \\
\hline-4 & RETORNOS & 1,168 & 828 &, 243 & ,00090614 &,- 00109741 & ,00290969 \\
\hline-3 & RETORNOS & ,693 & 827 & 488 & ,00051012 & -00138975 & 00240998 \\
\hline-2 & RETORNOS & 2,383 & 829 &, 017 & ,00182101 &,- 00015218 & ,00379420 \\
\hline-1 & RETORNOS & 2,653 & 831 & ,008 & 00245151 & ,00006628 & 00483675 \\
\hline 0 & RETORNOS & 2,669 & 836 & ,008 & ,00240800 & ,00007909 & ,00473691 \\
\hline 1 & RETORNOS & 4,812 & 828 &, 000 &, 00388429 & ,00180032 &, 00596826 \\
\hline 2 & RETORNOS & 1,593 & 825 &, 112 & 00123860 &,- 00076940 & ,00324661 \\
\hline 3 & RETORNOS & 1,001 & 827 &, 317 & 00077312 &,- 00122024 & ,00276647 \\
\hline 4 & RETORNOS &, 138 & 827 & 890 & 00011138 &,- 00197447 & ,00219722 \\
\hline 5 & RETORNOS &,- 636 & 833 &, 525 &,- 00046688 &,- 00236111 & ,00142735 \\
\hline
\end{tabular}

A Tabela 7 mostra que, com 99\% de confiança, rejeita-se a hipótese nula de que os retornos médios entre os dias -1, 0 e 1 são iguais a zero, e os retornos podem ser seguramente considerados como estatisticamente diferentes de zero e positivos, como se pode observar pelos , valores da estatística t de 2,653; 2,669 e 4,812, respectivamente, sendo que o valor crítico para aceitar a hipótese nula ao nível de significância estabelecido é de 2,575. Esses resultados mostram que, além da diferença estatística na análise realizada para a variância dos retornos médios entre os dias, em três dias da janela em estudos os retornos médios podem ser considerados como sendo significativamente diferentes de zero e positivos.

Para aprofundar os conhecimentos das estatísticas nas subamostras analisadas, aplicouse um teste t para cada subamostra analisada. $O$ resultado é apresentado na Tabela 8.

Tabela 8 - Teste t para subamostras

\begin{tabular}{|c|c|c|c|c|c|c|}
\hline & \multicolumn{6}{|c|}{ Test Value $=0$} \\
\hline & \multirow[t]{2}{*}{$\bar{T}$} & \multirow[t]{2}{*}{ Df } & \multirow[t]{2}{*}{ Sig. (2-tailed) } & \multirow[t]{2}{*}{ Mean Difference } & \multicolumn{2}{|c|}{$99 \%$ Confidence Interval of the Difference } \\
\hline & & & & & Lower & Upper \\
\hline VOLBAIXA & 1,278 & 289 & 202 & 0020 &,- 0020 &, 0060 \\
\hline VOLNORM & 1,389 & 303 & ,166 & 0020 &,- 0017 & ,0056 \\
\hline VOLALTA & 1,969 & 245 &, 050 &, 0035 &,- 0011 & 0080 \\
\hline DYMENOR & 1,851 & 576 & ,065 & 0020 &,- 0008 &, 0049 \\
\hline DYMAIOR & 1,863 & 268 & ,064 & 0029 &,- 0011 & 0070 \\
\hline
\end{tabular}

Da Tabela 8 nota-se que nenhuma subdivisão da base de dados ofereceu a possibilidade de retornos significativos e diferentes de zero com 99\% de confiança, pois o valor da estatística t não ultrapassou o valor crítico de 2,575 para poder ser considerado significativo.

\section{Conclusão}

Este trabalho procurou evidências de sinalização no anúncio do pagamento dos proventos das empresas constantes no índice Bovespa de janeiro a abril de 2006, proporcionando subsídios aos investidores marginais para a possibilidade da obtenção de retornos excessivos em datas pontuais e em uma janela de 11 dias ao redor das datas de anúncio. 
Os principais resultados encontrados para a amostra total em relação aos retornos anormais médios foram de 0,198, 0,195 e 0,345\%; e os acumulados, de 57,53, 59 ,33 e 84,93\%, para as subamostras, com baixa, normal e alta volatilidade, respectivamente.

$\mathrm{Na}$ análise de $-5 \mathrm{a}+5$, foram encontradas diferenças significativas para a variância média dos retornos anormais para a amostra total, ao nível de 5\% de significância, para os dias 1, 4 e 5 após o anúncio do pagamento dos proventos, no teste a posteriori. Esses resultados foram confirmados com a utilização do teste F, paramétrico; rejeitados pelo teste de Wilcoxon, não-paramétrico; e aceitos pelo teste de Kruskall-Wallis, não-paramétrico. No entanto, por consenso, foram adotados os resultados obtidos pelos testes paramétricos e de Kruskall-Wallis, nos quais se assumiu a conclusão de que houve diferença de variabilidade na variância média dos retornos anormais para os dias da janela em estudo.

A implicação desses resultados pode ser proveniente do conhecimento por parte dos investidores da presença de retornos excessivos ao redor das datas de anúncio, o que sugere que uma das possíveis causas dessa ocorrência é a de que os investidores já estejam realizando operações nessas datas na tentativa de obterem retornos excessivos.

Também foi encontrado que os retornos anormais podem ser considerados diferentes de zero e positivos para os dias -1, 0 e 1 para a amostra total, entretanto, para as diversas segmentações, os resultados não foram diferentes de zero.

Para a subamostra dividida de acordo com a volatilidade do mercado em que a data de anúncio ocorreu, os resultados mantiveram-se de acordo com o paradigma de risco e retorno, nos quais, para um mercado mais volátil ou de maior risco, obteve-se maiores retornos excessivos acumulados.

Na janela de 11 dias, de -5 a +5 dias ao redor da data da divulgação das informações, os resultados foram contrários aos esperados, encontrando-se retornos anormais médios e retornos anormais acumulados maiores para a amostra com baixa volatilidade. Esses resultados são opostos aos que se esperava com relação ao paradigma de risco x retorno, pois o mercado estaria operando com menor oscilação, portanto, menores riscos sinalizariam menores retornos.

Com relação à magnitude do dividendo pago, os resultados confirmaram as pesquisas anteriores já realizadas, sendo que a média dos retornos anormais para os dividendos com magnitude maior do que a média histórica foram superiores em relação aos retornos anormais obtidos para o pagamento de proventos menores do que a média histórica paga.

A hipótese nula de que não há retornos anormais foi rejeitada, e as hipóteses de que os investidores percebem a informação conduzida pelo pagamento dos dividendos de maneira simétrica em relação à volatilidade e ao dividend yield também foram rejeitadas, isto porque os resultados sugerem diferenças na obtenção dos retornos anormais, considerando-se essas características do mercado.

Dessa forma, tem-se a certeza de que este trabalho contribui para o aprofundamento da teoria da sinalização com relação à política de distribuição dos lucros. 


\section{Referências bibliográficas}

AGNES, M. P. Sinalização com dividendos em um ambiente sem impostos: evidências do Brasil. 2005, 153 f. Dissertação (Mestrado em Contabilidade e Finanças) - Escola de Administração - Programa de Pós-Graduação em Contabilidade e Finanças - Universidade Federal do Rio Grande do Sul, Porto Alegre, 2005.

ASQUITH, P.; MULLINS, D. W. The impact of initiating dividend payments on shareholder's wealth. Journal of Business, n. 56, v. 1, p. 7796, Jan. 1983.

BACKER, K. H.; VEIT, E. T.; POWELL, G. E. Factors influencing dividend policy decisions If NASDAQ firms. The Financial Review, n. 38, p. 19-38, 2001.

BERNARD, V. The feltham-ohlson framework: implications for empiricists. Contemporary Accounting Research, v. 11, n. 2, p. 733-747, Spring 1995.

BERNSTEIN, P. L. Dividends: the puzzle. Journal of Applied Corporate Finance, v. 9, n. 1, p. 1622, Spring 1996.

BONEAU, C. A. The effects of violations of assumptions underlying the t test. Psychological Bulletin, n. 57, v. 1, p. 49-64, Jan. 1960.

BRIEF, R. P.; ZAROWIN, P. The value relevance of dividends, book value and earnings. New York: University Dept. of Accounting Working Paper n. 99-3. Available at SSRN: < http:// ssrn.com/abstract $=173629$ or DOI: 10.2139 / ssrn.173629>. August 5, 1999.

BROWN, S. J.; WARNER, J. B. Measuring security price performance. Journal of Financial Economics, Amsterdan: North Holland, n. 8, v. 3, p. 205-258, Sept. 1980.

BURGSTAHLER, D.; DICHEV, I. Earnings, adaptation, an equity value. Washington: University of Washington, Aug. 1996.

COLLINS, D.; MAYDEW, E.; WEISS, I. Changes in the value-relevance of earnings and book values over the past forty years. Journal of Accounting and Economics, p. 39-67, 1997.
DECOURT, R.; PROCIANOY, J. L.; PIETRO NETO, J. As variações nas distribuições dos proventos em dinheiro sinalizam variações nos lucros futuros? In: EnANPAD, 31., 2007, Rio de Janeiro. Resumos... Rio de Janeiro, v. 1, p. 332332.

DOCKING, D. S.; KOCH, P. D. Sensitivity of investor reaction to market direction and volatility: dividend change announcements. The Journal of Financial Research, n. 28, v. 1, p. 21-40, Spring 2005.

FELTHAM, G.; OHLSON, J. Valuation and clean surplus accounting for operating and financial activities. Contemporary Accounting Research, v. 11, n. 2, p. 689-731, Spring, 1995.

FREIRE, H. V. L.; LIMA, I. S. O comportamento dos dividendos versus lucros anormais. In: ENCONTRO NACIONAL DE PÓSGRADUAÇÃO EM ADMINISTRAÇÃO, 27. 2003, Atibaia. Anais... Atibaia: EnANPAD, 2003. cd-rom.

FREIRE, H. V. L. et al. Dividendos e lucros anormais: um estudo nas empresas listadas na Bovespa. Revista de Contabilidade e Finanças, São Paulo, n. 39, p. 47-67, set./dez. 2005.

FIRMINO, A. L. G.; SANTOS, A. G. Q.; MATSUMOTO, A. S. Dividendos interessam? uma constatação empírica recente sobre a relevância da política de dividendos na bolsa de valores de São Paulo. In: ENCONTRO NACIONAL DE PÓS-GRADUAÇÃO EM ADMINISTRAÇÃO, 26, 2002, Salvador. Anais... Salvador: EnANPAD, 2002.cd-rom.

GORDON, M. J. Optimal investment and financing policy. Journal of Finance, n. 28, v. 2, p. 264-272. May 1963.

HAIR JR. et al. Análise multivariada de dados. 5. ed. Porto Alegre: Bookman, 2005.

$\mathrm{KOCH}$, A. S.; SUN, A. X. Dividend changes and the persistence of past earnings changes. The Journal of Finance, LIX(5), 2093-2116. (2004, October). 
KURONUMA, A. M.; LUCCHESI, E. P.; FAMÁ,

R. Retornos anormais acumulados das ações no período pós-pagamento de dividendos: um estudo empírico do mercado brasileiro. In: ENCONTRO NACIONAL DE PÓSGRADUAÇÃO EM ADMINISTRAÇÃO, 28., 2004, Curitiba. Anais... Curitiba: EnANPAD, 2004. cd-rom.

LINTNER, J. Distribution of income of corporations among dividends, retained earnings and taxes. American Economic Review, p.97113, May 1956.

MACKINLAY, C. Event studies in economics and finance. Journal of Economic Literature, n. 35, p. 13-39, March 1997.

MASULIS, R.W.; TRUEMAN, B. Corporate investment and dividend decisions under differential personal taxation. Journal of

Financial and Quantitative Analysis, n. 23, v. 4, p. 369-385, Dec. 1988.

MICHAELY, R.; ALLEN, F. Payout policy. The Wharton Financial Institutions Center, 1-148. (2002, April).

MODIGLIANI F.; MILLER, M. Dividend policy, growth and the valuation of share. Journal of Business, n. 34, p. 411-433, Oct. 1961.

MOUGOUÉ, M.; RAO, R. P. The information signaling hypothesis of dividends: evidence from cointegration and causality tests. Journal of Business Finance e Accounting, v. 30, Apr./May 2003.
NOVIS, J. A. N.; SAITO, R. Pagamentos de dividendos e persistência de retornos anormais das ações: evidência do mercado brasileiro. In: ENCONTRO NACIONAL DE PÓSGRADUAÇÃO EM ADMINISTRAÇÃO, 27. 2003, Atibaia. Anais... Atibaia: EnANPAD, 2003. cd-rom.

OHLSON, J. Earnings, book values and dividends in equity valuation. Contemporary Accounting Research, v. 11, n. 2, p. 661-687, Spring 1995.

PETTIT, R. Dividend announcements, security performance, and capital market efficiency. Journal of Finance, n. 27, p. 993-1007, Dec. 1972.

PROCIANOY, J. L.; VERDI, R. O efeito clientela no mercado brasileiro: será que os investidores são irracionais? Revista Brasileira de Finanças, n. 1, v. 2, p. 22-35, 2003.

STRONG, N. C. Discussion of the impact of dividends, debt, and investment on valuation models. Journal of Business Finance \& Accounting, n. 24, v. 7-8, p. 1141-1145, 1997.

STRONG, N. C.; WALKER, M.; HARDING, Z.

Price: models and earnings response coefficients. Manchester: University of Manchester, 1996.

REES, W. P. The Impact of dividends, debt and investment on valuation model's. Journal of Business Finance \& Accounting, 1997.

VERONESI, P. Stock market overreaction to bad news in good times: a rational expectations equilibrium model. The Review of Financial Studies, Winter, 1999. 